\title{
ON THE OSCILLATION OF CERTAIN THIRD ORDER LINEAR DIFFERENTIAL EQUATIONS
}

\author{
G. J. ETGEN AND C. D. SHIH
}

\begin{abstract}
This paper is concerned with the relationship between the concepts of oscillation, nonoscillation and disconjugacy of the general third order linear differential equation $y^{\prime \prime \prime}+p(x) y^{\prime \prime}+q(x) y^{\prime}+r(x) y=0$. Two cases are considered: (i) $q(x)$ nonpositive and $r(x)$ nonnegative and (ii) both $q(x)$ and $r(x)$ nonpositive. Sufficient conditions for the disconjugacy of the equation are presented, improving the recent work of W. J. Kim. The relationship between disconjugacy and oscillation is described in each case, and these results provide a partial answer to a question raised by $\mathrm{J}$. $\mathrm{H}$. Barrett.
\end{abstract}

1. Introduction. This paper is concerned with third order linear differential equations of the form

$$
y^{\prime \prime \prime}+p(x) y^{\prime \prime}+q(x) y^{\prime}+r(x) y=0
$$

where $p(x), q(x)$, and $r(x)$ are continuous functions on the interval $[a, \infty)$. Our interest is in the relationship between the concepts of oscillation and disconjugacy of (1). In particular, we shall say that (1) is oscillatory if the equation has a nontrivial solution $y(x)$ such that $y(x)$ has infinitely many zeros on $[a, \infty)$. On the other hand, if there exists a number $b, b \geqq a$, such that no nontrivial solution of (1) has more than two zeros, counting multiplicities, on $[b, \infty)$, then (1) is said to be disconjugate on this interval. Clearly, if (1) is disconjugate on some interval $[b, \infty), b \geqq a$, then (1) is nonoscillatory on $[a, \infty)$, i.e., no nontrivial solution of (1) has more than a finite number of zeros on $[a, \infty)$. In the case of the second order differential equation

$$
\left(f(x) y^{\prime}\right)^{\prime}+g(x) y=0
$$

where $f(x)$ and $g(x)$ are continuous functions and $f(x)>0$ on $[a, \infty)$, the relationship between the concepts described above is an easy consequence of the Sturm separation theorem. Specifically, (2) is nonoscillatory

Received by the editors September 1, 1972 and, in revised form, March 9, 1973.

AMS (MOS) subject classifications (1970). Primary 34C10.

Key words and phrases: Third order linear differential equations, disconjugacy, oscillation of solutions. 
on $[a, \infty)$ if and only if there is a number $b, b \geqq a$, such that (2) is disconjugate on $[b, \infty)$. Alternatively, (2) is oscillatory if and only if (2) is not disconjugate on $[c, \infty)$ for each $c \geqq a$. Since the Sturm separation theorem does not carry over to the general third order equation (1), the relationship between the concepts of oscillation, nonoscillation and disconjugacy is more complicated than in the second order case.

In this paper, we are concerned with equation (1) in the two cases distinguished by the following hypotheses on the coefficients $q(x)$ and $r(x)$ :

$\left(\mathrm{H}_{1}\right) q(x) \leqq 0, r(x) \geqq 0$ and $q^{2}(x)+r^{2}(x) \not \equiv 0$ on any subinterval of $[a, \infty)$.

$\left(\mathrm{H}_{2}\right) q(x) \leqq 0, r(x) \leqq 0$ and $q(x)+r(x) \not \equiv$ on any subinterval of $[a, \infty)$.

In each of the cases we establish conditions which imply that (1) is disconjugate on some subinterval $[b, \infty)$ of $[a, \infty)$. Our sufficient conditions for the disconjugacy of (1) improve the results of W. J. Kim [7, Theorems 4 and 7]. Assuming that (1) is not disconjugate on any subinterval $[c, \infty)$ of $[a, \infty)$, we show, in each case, that (1) is actually oscillatory. This work provides a partial answer to the question raised by J. H. Barrett in [2, p. 213]. There are examples of third order differential equations which are not disconjugate on any subinterval $[b, \infty)$ of $[a, \infty)$ and yet are nonoscillatory (see Barrett [2, p. 213] and M. Dolan [4, p. 385].

In order to relate the nondisconjugacy of $(1)$ on any subinterval $[b, \infty)$ of $[a, \infty)$ with the existence of an oscillatory solution of (1) we shall require the following theorem which is a combination of the results of Barrett [3, Theorem 2.15] and Dolan [4, Theorem 1].

THEOREM A. If the differential equation (1) is nonoscillatory on $[a, \infty)$, then either all solutions of the adjoint

$$
z^{\prime \prime \prime}-(p(x) z)^{\prime \prime}+(q(x) z)^{\prime}-r(x) z=0
$$

of (1) are oscillatory, or all solutions of $\left(1^{*}\right)$ are nonoscillatory. Moreover, if each of (1) and $\left(1^{*}\right)$ is nonoscillatory on $[a, \infty)$, then each equation is disconjugate on some subinterval $[b, \infty)$ of $[a, \infty)$.

2. Case I. In this section we assume that the coefficients satisfy hypothesis $\left(\mathrm{H}_{1}\right)$. Our first theorem is a sufficient condition that (1) be disconjugate on some subinterval $[b, \infty)$ of $[a, \infty)$. Our result improves a corresponding result of $\operatorname{Kim}[7$, Theorem 4].

THEOREM 1. Let the coefficients of (1) satisfy $\left(\mathrm{H}_{1}\right)$, and let $p(x) \in$ $C^{\prime \prime}[a, \infty)$ and $q(x) \in C^{\prime}[a, \infty)$. If there exists a number $b, b \geqq a$, such that $q(x)-p^{\prime}(x) \leqq 0$ and $r(x)-q^{\prime}(x)+p^{\prime \prime}(x) \leqq 0$ on $[b, \infty)$, then (1) is disconjugate on $[b, \infty)$. 
Remark. In addition to the hypothesis of the theorem, Kim [7, Theorem 4] assumes that $p(x)$ is of one sign and $q(x)-2 p^{\prime}(x) \leqq 0$. Since $q(x)-2 p^{\prime}(x) \leqq 0$ implies $q(x)-p^{\prime}(x) \leqq 0$, our inequality is "weaker" than Kim's inequality.

Proof. Let $b, b \geqq a$, be a number such that the inequalities $q-p^{\prime} \leqq 0$ and $r-q^{\prime}+p^{\prime \prime} \leqq 0$ hold on $[b, \infty)$. Let $P(x)=\exp \int_{a}^{x} p(t) d t$ and multiply (1) by $P(x)$ to obtain

$$
\left(P(x) y^{\prime \prime}\right)^{\prime}+Q(x) y^{\prime}+R(x) y=0,
$$

where $Q(x)=P(x) q(x)$ and $R(x)=P(x) r(x)$. Since $P(x)>0$ on $[a, \infty)$, $Q(x) \leqq 0$ and $R(x) \geqq 0$ on this interval. It is clear that (1) and (3) have the same behavior with respect to disconjugacy, nonoscillation and oscillation.

Using an immediate extension of a result of Lazer [8, Lemma 1.1], it is easily seen that if $y(x)$ is a solution of (3) satisfying the conditions

$$
y(c) \geqq 0, \quad y^{\prime}(c) \geqq 0, \quad y^{\prime \prime}(c)>0
$$

for any point $c, c>a$, then $y(x)>0, y^{\prime}(x)<0$ and $y^{\prime \prime}(x)>0$ on $[a, c)$. In particular, if $y(x)$ has a double zero at $x=c, c>a$, then $y(x) \neq 0$ on $[a, c)$. A third order differential equation having this property is said to be $(1,2)$-nonoscillatory (see [1, p. 233] and [5, p. 921]). Similarly, equation (3) (or, equivalently, (1)) is said to be $(2,1)$-nonoscillatory if each nontrivial solution $y(x)$ having a double zero does not vanish to the right of the double zero.

It is well known $[1$, p. 239] that (1) is disconjugate on $[b, \infty)$ if and only if $(1)$ is both $(1,2)$ and $(2,1)$-nonoscillatory. Since $(3)$ is $(1,2)$-nonoscillatory, $(1)$ is $(1,2)$-nonoscillatory, and thus it remains to show that (1) is $(2,1)$-nonoscillatory. Let $u(x)$ be the solution of (1) satisfying the initial conditions:

$$
u(b)=u^{\prime}(b)=0, \quad u^{\prime \prime}(b)=1 .
$$

Since $u^{\prime \prime}(b)=1>0, u^{\prime}(x)>0$ on some interval $(b, b+\delta), \delta>0$. Suppose $u^{\prime}(x)$ has a zero on $(b, \infty)$ and let $x=d$ be the first such zero. Integrating (1) from $b$ to $t, b<t<d$, we have

$$
\begin{aligned}
& u^{\prime \prime}(t)+p(t) u^{\prime}(t)-\left[p^{\prime}(t)-q(t)\right] u(t) \\
& \quad+\int_{b}^{t}\left[p^{\prime \prime}(s)-q^{\prime}(s)+r(s)\right] u(s) d s=1 .
\end{aligned}
$$

Since $u(x)>0$ on $(b, d)$, we have, upon letting $t \rightarrow d^{-}$and using the inequalities stated in our theorem, $u^{\prime \prime}(d) \geqq 1$. However, since $u^{\prime \prime}(b)=1$ and $d$ is the first zero of $u^{\prime}(x)$ to the right of $x=b, u^{\prime \prime}(d) \leqq 0$ and we have a contradiction. We conclude, therefore, that $(1)$ is $(2,1)$-nonoscillatory on $[b, \infty)$ and our theorem follows. 
Our next theorem relates nondisconjugacy of (1) on any subinterval $[b, \infty)$ of $[a, \infty)$ with the oscillation of (1). Our result answers the question raised by Barrett [2, p. 213] in this case.

THEOREM 2. Let the coefficients of (1) satisfy $\left(\mathrm{H}_{1}\right)$. If for each number $b, b \geqq a$, there is a nontrivial solution of (1) having a least three zeros on $[b, \infty)$, then (1) is oscillatory.

Proof. Suppose (1) is nonoscillatory. Then, by Theorem A, all nontrivial solutions of the adjoint $\left(1^{*}\right)$ of $(1)$ are oscillatory, i.e. $\left(1^{*}\right)$ is strongly oscillatory.

We conclude from our proof of Theorem 1 and the fact that (1) and (3) are equivalent with respect to oscillatory behavior, that $(1)$ is $(1,2)-$ nonoscillatory. Thus, from [1, Lemma 1, p. 237], $\left(1^{*}\right)$ is $(2,1)$-nonoscillatory, i.e. if $z(x)$ is a solution of $\left(1^{*}\right)$ having a double zero at some point $c, c \geqq a$, then $z(x) \neq 0$ on $(c, \infty)$. This contradicts the fact that $z(x)$ is oscillatory.

The converse of Theorem 2 namely: if (1) is oscillatory, then for each number $c, c \geqq a$, there is a nontrivial solution of (1) having at least three zeros on $[c, \infty)$, clearly holds. Conditions which imply that (1) is oscillatory have been established by A. C. Lazer [8, Theorem 1.3].

3. Case II. In this section we assume that the coefficients satisfy hypothesis $\left(\mathrm{H}_{2}\right)$. Our first theorem provides a sufficient condition that (1) be disconjugate on some subinterval $[b, \infty)$ of $[a, \infty)$. This result improves the result of $\mathrm{Kim}$ [7, Theorem 7] and can be obtained using his proof together with the fact that (1) and (3) are equivalent with respect to oscillation.

THEOREM 3. Let the coefficients of (1) satisfy $\left(\mathrm{H}_{2}\right)$. If there is a number $b, b \geqq a$, such that $q(x) \in C^{\prime}[b, \infty)$ and $p(x) q(x)+q^{\prime}(x)-r(x) \leqq 0$ on $[b, \infty)$, then (1) is disconjugate on $[b, \infty)$.

REMARK. In addition to the hypothesis of our theorem, Kim [7, Theorem 7] assumes $p(x) \in C^{\prime}[b, \infty), p(x) \leqq 0$ and $p^{2}(x)+p^{\prime}(x)+q(x) \leqq 0$.

Our final theorem shows that if (1) is not disconjugate on any subinterval $[b, \infty)$ of $[a, \infty)$, then (1) is oscillatory, answering Barrett's question in this case.

THEOREM 4. Let the coefficients of (1) satisfy $\left(\mathrm{H}_{2}\right)$. If for each number $b, b \geqq a$, there is a nontrivial solution of (1) having at least three zeros on $[b, \infty)$, then (1) is oscillatory.

Proof. Multiply (1) by $P(x)=\exp \int_{a}^{x} p(t) d t$ to obtain (3). Since $q(x)$ and $r(x)$ are nonpositive on $[a, \infty), Q(x)=P(x) q(x)$ and $R(x)=$ $P(x) r(x)$ are nonpositive on this interval, and from these sign assumptions 
it is easily verified that (3), and therefore (1), is $(2,1)$-nonoscillatory. We can now conclude that the adjoint $\left(1^{*}\right)$ of $(1)$ is $(1,2)$-nonoscillatory [1, Lemma 1, p. 237].

Suppose (1) is nonoscillatory. By hypothesis, (1) is not disconjugate on any subinterval $[b, \infty)$ of $[a, \infty)$, and so $\left(1^{*}\right)$ must be strongly oscillatory by Theorem $\mathrm{A}$. However, since $\left(1^{*}\right)$ is $(1,2)$-nonoscillatory, we can use the sequence argument employed by Lazer [8, Theorem 1.1] to construct a nontrivial solution $w(x)$ of $\left(1^{*}\right)$ such that $w(x) \geqq 0$ on $[a, \infty)$. Thus, all zeros of $w(x)$ must be double zeros. But again using the fact that $\left(1^{*}\right)$ is $(1,2)$-nonoscillatory, we conclude that $w(x)$ has at most one zero and we have a contradiction.

\section{REFERENCES}

1. N. Y. Azbelev and Z. B. Calyuk, On the question of distribution of zeros of solutions of linear differential equations of the third order, Mat. Sb. 51 (93) (1960), 475-486; English transl., Amer. Math. Soc. Transl. (2) 42 (1964), 233-245. MR 22 \#12266.

2. J. H. Barrett, Third order differential equations with nonnegative coefficients, J. Math. Anal. Appl. 24 (1968), 212-224. MR 38 \#365.

3. - Oscillation theory of ordinary linear differential equations, Associated Western Universities Differential Equations Symposium, Boulder, Colorado, 1967, Lectures on Ordinary Differential Equations (edited by Robert McKelvey), Academic Press, New York, 1970.

4. J. M. Dolan, On the relationship between the oscillatory behavior of a linear third order differential equation and its adjoint, J. Differential Equations 7 (1970), 367-388. MR 41 \#568.

5. M. Hanan, Oscillation criteria for third order linear differential equations, Pacific J. Math. 11 (1961), 919-944. MR 26 \#2695.

6. P. Hartman, Ordinary differential equations, Wiley, New York, 1964. MR 30 $\# 1270$.

7. W. J. Kim, Oscillatory properties of linear third order differential equations, Proc. Amer. Math. Soc. 26 (1970), 286-293. MR 41 \#8758.

8. A. C. Lazer, The behavior of solutions of the differential equation $y^{\prime \prime \prime}+p(x) y^{\prime}+$ $q(x) y=0$, Pacific J. Math. 17 (1966), 435-466. MR 33 \#1552.

Department of Mathematics, University of Houston, Houston, Texas 77004 